\title{
Combined segregation and linkage analysis of HLA markers in familial psoriasis
}

\author{
Sun-Wei Guo ${ }^{1}$, Stefan Jenisch ${ }^{2}$, Philip Stuart ${ }^{3}$, Ethan M Lange ${ }^{4}$, Debra Kukuruga ${ }^{5}$, \\ Rajan Nair ${ }^{3}$, Tilo Henseler ${ }^{6}$, John Voorhees ${ }^{3}$, Enno Christophers ${ }^{6}$ and James T Elder*3,7,8
}

${ }^{1}$ Department of Pediatrics, Medical College of Wisconsin, Wisconsin, USA; ${ }^{2}$ Department of Immunology, University of Kiel, Kiel, Germany; ${ }^{3}$ Department of Dermatology, University of Michigan Medical School, Ann Arbor, Michigan, USA; ${ }^{4}$ Department of Public Health Sciences, Section on Biostatistics, Wake Forest University School of Medicine, Winston-Salem, North Carolina, USA; ${ }^{5}$ Bone Marrow Transplantation Program, Wayne State University, Detroit, Michigan, USA; ${ }^{6}$ Department of Dermatology, University of Kiel, Kiel, Germany; ${ }^{7}$ Department of Radiation Oncology (Cancer Biology), University of Michigan Medical School, Ann Arbor Michigan, USA; ${ }^{8}$ Ann Arbor Veterans Affairs Hospital, Ann Arbor, Michigan, USA

Marker-based segregation analysis (MBSA) is a modification of a published method of combined linkage and segregation analysis (Am J Hum Genet 51: 1111 -1126, 1992), to determine whether a candidate gene known to be associated with the disease of interest is truly segregating with the disease in families. Here we outline the conceptual basis of MBSA and present a Monte Carlo method for significance testing. The method is applied to PSORS1, a locus within the major histocompatibility complex (MHC) for which linkage and linkage disequilibrium with psoriasis has already been demonstrated. The results are very consistent with our current knowledge of PSORS1, and suggest that MBSA can provide useful information on genotype-phenotype relationships such as penetrance and allelic heterogeneity.

European Journal of Human Genetics (2002) 10, 327-333. doi:10.1038/sj.ejhg.5200810

Keywords: linkage analysis; segregation analysis; Monte Carlo methods; human leukocyte antigens; major histocompatibility complex; psoriasis

\section{Introduction}

The complex genetic disorders remain as a formidable challenge in human genetics. While a dense map of genetic markers exists and a substantial repertoire of analytical methods have been developed, progress in the identification of disease genes in multifactorial diseases lags behind that obtained in monogenic disorders. ${ }^{1,2}$ Because many of the socalled complex genetic disorders are relatively common, study cohort sizes continue to increase. Given the steady increase in resolution of genetic maps and in the informativeness of their component markers, we can expect that an increasing number of candidate regions will be subjected to

*Correspondence: James T Elder, 3312 CCGC, Box 0932, University of Michigan, Ann Arbor, MI 48109-0932, USA. Tel: (734) 763-0355; Fax: (734) 763-4575; E-mail: jelder@umich.edu Received 3 August 2000; revised 25 March 2002; accepted 4 April 2002 saturation genotyping for the purpose of linkage disequilibrium mapping.

Allelic association studies are more powerful than affected sib-pair methods for disease gene localization when linkage disequilibrium is present. ${ }^{3,4}$ To avoid the confounding effects of population stratification that may arise in case-control studies, ${ }^{5}$ pedigree-based tests of linkage disequilibrium such as the transmission/disequilibrium test (TDT) have been developed. ${ }^{6,7}$ However, there are drawbacks to pedigreebased tests for allelic association. The TDT uses data collected from cases and their parents. Larger pedigrees have to be broken up in order to perform the TDT, thereby restricting the available information on allele transmission to that provided by the nuclear family. This strategy does not optimally utilise the additional information on gene transmission and genotype-phenotype relations that is present in large pedigrees. This problem has been mitigated to some extent with the development of the pedigree 
disequilibrium test (PDT). ${ }^{8,9}$ However, the PDT does not determine whether the gene under study acts in a dominant or a recessive fashion. In order to develop a tool that might more completely assess the relationship of genotype and phenotype, we have developed a method called marker-based segregation analysis (MBSA). MBSA is an extension of a previously-published method for combined linkage and segregation analysis originally developed by one of us. ${ }^{10}$ Here we outline the theoretical basis of MBSA, present a Monte Carlo method for evaluating the significance of the results, and utilize the method to analyse HLA marker data collected from multiplex families manifesting psoriasis, an inflammatory and hyperproliferative skin disease. ${ }^{11,12}$

It has long been appreciated that psoriasis displays strong familial aggregation, with one of the highest heritabilities of any complex genetic disorder $(60-90 \%)^{13}$ and sibling recurrence risks $\lambda_{\mathrm{s}}$ of approximately $10 .{ }^{14}$ Genome scans have provided suggestive evidence for linkage of psoriasis to HLA as well as several non-HLA loci; ${ }^{15-20}$ the HLA-linked locus has been designated PSORS1. ${ }^{21}$ However, some of these scans found no evidence for linkage to HLA. ${ }^{15,16,20}$ We subsequently showed that the evidence for linkage to HLA is markedly increased when linkage disequilibrium information is taken into account. ${ }^{11,12}$ Recently, PSORS1 has been fine-mapped to the vicinity of HLA-C by linkage disequilibrium mapping. ${ }^{22,23}$ We have further refined this interval to an approximately $60 \mathrm{~kb}$ interval residing 30 to $90 \mathrm{~kb}$ telomeric to HLA-C (MHC). ${ }^{24}$

Here we demonstrate that MBSA yields highly significant evidence for segregation of alleles at several HLA loci with psoriasis, whether controls are drawn from the founders of the ascertained population or from local controls. Segregation parameters were strongly dependent on the control population used. The strongest evidence for segregation was found for HLA-C, consistent with our existing knowledge of the location of PSORS1. However, the results of this analysis do not confirm or exclude a role for HLA-C as the causative gene.

\section{Subjects and Methods}

All studies were approved by the Human Subjects Committees of the Institutional Review Boards of the University of Michigan and the University of Kiel. Probands for the pedigree studies were identified through clinic records or encounters, via advertising, or in response to media reports. Psoriasis was defined by the presence of at least two characteristic skin lesions, ${ }^{25}$ or by a single lesion occupying greater than $1 \%$ of the total body surface area. Ascertainment was for juvenile onset psoriasis in the proband (i.e., age at onset $\leqslant 40$ years $^{26}$ ). No age of onset criterion was applied to other family members. After obtaining informed consent, all participants received a total body skin examination and provided a blood sample. In order to estimate allele frequencies in the general population, we also typed all five HLA loci in two cohorts of unrelated local controls (189 US and 124 German). All of these cohorts have been described. ${ }^{11}$ Some of their characteristics are presented in Table 1.

HLA-A and HLA-B were typed serologically as described. ${ }^{27}$ HLA-C typing was performed by PCR using sequence-specific primers. ${ }^{28}$ HLA-DRB1 and HLA-DQB1 were typed by groupspecific PCR followed by hybridization to sequence-specific oligonucleotide probes, according to the Eleventh Histocompatibility Workshop reference protocol. ${ }^{29}$

To carry out the MBSA, the (unknown) marker alleles were grouped into two risk classes, termed 'risk' and 'non-risk', with all members of a class constrained to carry identical risk (Figure 1). Three parameters were used to describe the penetrances of the resulting genotypes ('risk/risk', 'risk/nonrisk', and 'non-risk/non-risk'). Let $\mathbf{M}$ denote the observed marker phenotype vector, $X$ the vector of disease status $\left(\mathrm{X}_{\mathrm{i}}=1\right.$ if the $\mathrm{i}$-th individual is affected or 0 otherwise), and $\mathrm{G}$ the vector of true underlying marker genotypes. Then, the likelihood of observing $\mathrm{M}$ and $\mathrm{X}$ is:

$$
\begin{aligned}
& \mathrm{L}(\mathrm{X}, \mathrm{M})=\sum_{G} P(X \mid G) P(M \mid G) P(G) \\
& =\sum_{G}\left(\prod_{i \in F} P\left(G_{i}\right) \prod_{j \in F} P\left(G_{j} \mid G_{j f}, G_{j m}\right) \prod_{k} P\left(X_{k} \mid G_{k}\right) P\left(M_{k} \mid G_{k}\right)\right)
\end{aligned}
$$

where $F$ is the set of all founders, i, j, and $k$ are indices referring to founders, non-founders, and all individuals, respectively, $P\left(G_{i}\right)$ is the frequency of founder genotype $G_{i}$, $P\left(G_{j} \mid G_{j f}, G_{j m}\right)$ is the transmission probability of nonfounder genotype $G_{j}$ given paternal genotype $G_{j f}$ and maternal genotype $G_{j m}, P\left(M_{k} \mid G_{k}\right)$ is the probability of observing $M_{k}$ given $G_{k}$, and $P\left(X_{k} \mid G_{k}\right)$ is the penetrance probability for genotype $G_{k}$, and the summation is over all possible genotypic configurations. The overall likelihood is the

\begin{tabular}{|c|c|c|}
\hline Multiplex families & US & German \\
\hline Number of extended pedigrees & 16 & 22 \\
\hline Number of nuclear sib pair families & 33 & 16 \\
\hline Proportion of males affected & $\begin{array}{c}79 / 182 \\
(43.4 \%)\end{array}$ & $\begin{array}{c}66 / 159 \\
(41.5 \%)\end{array}$ \\
\hline Proportion of females affected & $\begin{array}{c}97 / 223 \\
(43.5 \%)\end{array}$ & $\begin{array}{c}77 / 184 \\
(41.8 \%)\end{array}$ \\
\hline Proportion of all individuals affected & $\begin{array}{l}176 / 405 \\
(43.5 \%)\end{array}$ & $\begin{array}{l}143 / 343 \\
(41.7 \%)\end{array}$ \\
\hline Proportion of parents affected ${ }^{b}$ & $\begin{array}{c}42 / 112 \\
(37.5 \%)\end{array}$ & $\begin{array}{c}31 / 70 \\
(44.3 \%)\end{array}$ \\
\hline One parent affected (\%) & $42(75 \%)$ & $23(65.7 \%)$ \\
\hline Neither parent affected (\%) & $14(25 \%)$ & $8(22.9 \%)$ \\
\hline Both parents affected (\%) & $0(0 \%)$ & $4(11.4 \%)$ \\
\hline \multicolumn{3}{|l|}{ Local controls } \\
\hline Source of blood sample & $\begin{array}{c}\text { Potential } \\
\text { organ donors }\end{array}$ & $\begin{array}{l}\text { Blood } \\
\text { donors }\end{array}$ \\
\hline Number of individuals typed & 189 & 124 \\
\hline
\end{tabular}

Table 1 Selected characteristics of the study cohorts ${ }^{\mathrm{a}}$ 


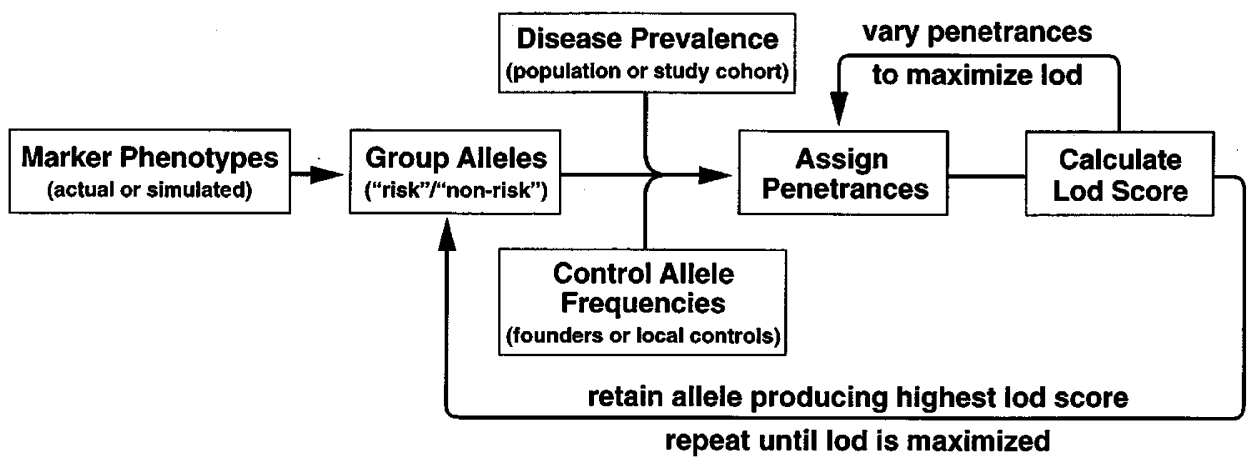

Figure 1 Marker-based segregation analysis flow chart. See Subjects and Methods for details of method.

product of the likelihoods of all individual pedigrees. The HLA typing data were considered to represent phenotypes, rather than genotypes, because this makes the method more robust to missing data. For instance, an HLA phenotype of Cw6 is consistent with two underlying genotypes: Cw6/Cw6 and Cw6/blank. This feature was particularly useful in this analysis because HLA-C blank alleles are commonly encountered $^{30}$ and because the number of usable HLA phenotypes varied from locus to locus.

Under the null hypothesis of independent segregation of marker and disease alleles, the probability of being affected is a constant, set equal to the prevalence of psoriasis for all genotypes. Specific disease models were embodied by the genotypes comprising the risk class and the penetrance parameters for each of the three genotype classes. These models were tested against the model under the null hypothesis using the $\log _{10}$ likelihood ratio:

$\log _{10}[\mathrm{~L}(\mathrm{X}, \mathrm{M} \mid$ specification of penetrance $) \div$ $\mathrm{L}(\mathrm{X}, \mathrm{M} \mid$ same penetrances for all genotypes $)]$.

Computation of the likelihood was carried out under the Elston-Stewart algorithm, ${ }^{31}$ using a modified version of MENDEL. ${ }^{32}$ Disease models were systematically tested by optimizing the lod score over a variety of allele combinations. To accomplish this, a program called GEN3 was developed to iteratively maximize the LOD score. The program first identifies the single best allele, then adds additional alleles to the risk group until the LOD score no longer increases. For each collection of risk alleles, a modification of the APEN subroutine of the attached program SEGV varies the penetrance parameters iteratively until the maximal lod score is obtained, under the constraint that the sum of the products of the penetrance and frequency of each genotype class must equal the user-specified disease prevalence (program parameter CVALUE(1) in subroutine INITIAL). This procedure is depicted in Figure 1.

Statistical significance was determined using a Monte Carlo approach. Consider a control population that matches the study group in terms of pedigree structure, affectation status and allele distribution in founders, but in which the marker and trait do not co-segregate. Because the method iterates the penetrances and retains the penetrances producing the highest lod score, the expectation of the MBSA lod score is positive for such a population even when a single allele is placed in the risk group. In addition, multiple tests are performed to determine the optimal set of risk alleles, meaning that the expectation of the lod score will be even more positive. However, the actual distribution of lod scores under the null hypothesis is unknown. Therefore, we evaluated statistical significance using genotype simulations. To accomplish this, 99 sets of simulated genotypes were generated using SIMLINK, ${ }^{33}$ and MBSA lod scores were calculated. When the observed genotypes yielded a lod score falling within the top $5 \%$ of all 100 tests, the results were deemed significant.

Ascertainment correction was made by conditioning on proband disease phenotype. However, complete ascertainment correction was not possible, due to the diversity of family structures sampled, and to the deliberate recruitment of sib pair and multiplex families. The selection of appropriate allele frequencies to use in this analysis therefore posed a challenge. These frequencies could be estimated based on the founders of the ascertained sample, or by a geographically-matched population sample. As previously reported, ${ }^{12}$ these two estimates differ substantially due to ascertainment bias. Moreover, the denominator of the likelihood ratio depends on the assumed prevalence of psoriasis, which is much greater in our sample $(42.6 \%)$ than in the general population $(1-2.8 \%)$. $^{34}$

To empirically assess the effects of these variables on the results of MBSA, the Monte Carlo analysis described above was perfomed twice. In the first analysis (termed 'populationbased'), the parameter CVALUE(1) in the subroutine INITIAL was set to 0.02 , corresponding to a disease prevalence of $2 \%$, and marker allele frequencies were derived from the local controls. In the second analysis (termed 'sample-based'), CVALUE(1) was set to 0.426 , corresponding to a disease prevalence of $42.6 \%$, and the marker allele frequencies were derived from founders. When no individual in a control sample carried an allele found in the affected sample, a 
control allele frequency of $1 / 2 n$ was assumed, where $n$ is the total number of individuals sampled.

\section{Results}

As expected in segregation analysis, ${ }^{35}$ MBSA proved to be quite sensitive to the choice of marker and disease allele frequencies. Much higher lod scores were obtained at all loci tested under the population-based method (Table 2); however, statistically significant results were obtained for HLA-C in both cohorts under the sample-based method as well (Table 2 and Figure 2). The most prominent contributions were made by HLA-Cw6, HLA-B57, HLA-DRB*0701 (DR7) and HLA-DQB1*03032 (DQ9), all components of the ancestral HLA haplotype known as EH57.1. ${ }^{36}$ This ancestral haplotype is known to be markedly increased in frequency in psoriasis (Table 2). ${ }^{11,12,37}$ However, other alleles also contributed to the final lod scores, as shown graphically for HLA$\mathrm{B}$ and HLA-C in Figure 3. Most of the $\mathrm{B}$ alleles identified by MBSA corresponded to HLA-B/-C haplotypes known to carry Cw6. ${ }^{24,30}$ These included several $\mathrm{B}$ alleles not previously known to be psoriasis-associated (e.g., B45 and B50 in the German cohort, and B47 in the US cohort).

The magnitudes of the penetrance parameters could not be meaningfully compared between the population-based and sample-based methods, as they reflect markedly different expectations under the null hypothesis. However, under the population-based method, possession of one risk allele was associated with a substantial increase in penetrance over the null value of 0.02 (defined by the general population prevalence of $2 \%$ ). Under the sample-based method, two risk alleles were necessary to increase the null penetrance of 0.43 dictated by the $42.6 \%$ disease prevalence in our sample (Table 2 ).

\section{Discussion}

This is the first description of MBSA, a novel extension of a previously-described method of combined segregation and linkage analysis. ${ }^{10} \mathrm{MBSA}$ allows maximal extraction of genetic information from partially-typed pedigrees, and minimizes the loss of information due to homozygosity at the disease locus. Furthermore, it provides a framework in which various genotype-phenotype relationships can be assessed systematically. George and Elston proposed a similar method to determine whether a significant portion of the variability of a quantitative trait can be attributed to segregation at a known marker locus. ${ }^{38}$ However, this is the first time that such a method has been applied to a qualitative trait.

The TDT is more computationally efficient than MBSA, especially if significance testing is included. Moreover, the TDT does not require specification of control allele frequencies or disease prevalence. Therefore, we do not recommend MBSA as a replacement for the TDT. However, under conditions of controlled ascertainment, MBSA holds promise for the prediction of genotype-phenotype relationships, including the definition of penetrances for different genotype classes and the capacity to identify multiple founder events (allelic heterogeneity). We will first consider this potential advantage of MBSA, then move on to interpret our results in the context of what is currently known about PSORS1.

Table 2 MBSA results

\begin{tabular}{|c|c|c|c|c|c|c|c|}
\hline Cohort & HLA locus & lod score & Rank & $\begin{array}{c}\text { Penetrance } \\
\text { risk/risk }\end{array}$ & $\begin{array}{l}\text { Penetrance } \\
\text { risk/nonrisk }\end{array}$ & $\begin{array}{c}\text { Penetrance } \\
\text { nonrisk/nonrisk }\end{array}$ & Risk alleles ${ }^{c}$ \\
\hline \multicolumn{8}{|c|}{ A. Population-based method ${ }^{a}$} \\
\hline German & A & 22.2 & 1 & 0.05 & 0.01 & 0.002 & $2,1,30,24,10$ \\
\hline German & C & 66.7 & 1 & 0.44 & 0.08 & 0.005 & $6,1,15,14$ \\
\hline German & B & 55.4 & 1 & 0.21 & 0.08 & 0.005 & $57,45,52,13,50,37$ \\
\hline German & DRB1 & 24.0 & 1 & 0.07 & 0.02 & 0.006 & ${ }^{*} 0701,{ }^{*} 13,{ }^{*} 0301,{ }^{*} 14$ \\
\hline German & DQB1 & 17.2 & 1 & 0.05 & 0.07 & 0.01 & *03032 \\
\hline US & $\mathrm{C}$ & 33.0 & 1 & 0.34 & 0.05 & 0.009 & 6,1 \\
\hline US & B & 22.5 & 1 & $0.50^{\mathrm{b}}$ & 0.09 & 0.01 & $57,37,47$ \\
\hline US & DRB1 & 15.9 & 2 & 0.15 & 0.03 & 0.01 & *0701, *1001 \\
\hline US & DQB1 & 15.7 & 1 & 0.31 & 0.06 & 0.01 & ${ }^{\star} 03032,{ }^{\star} 0302$ \\
\hline \multicolumn{8}{|c|}{ B. Sample-based method ${ }^{\mathrm{d}}$} \\
\hline German & C & 6.4 & 1 & 0.83 & 0.58 & 0.30 & $6,15,13$ \\
\hline US & $\mathrm{C}$ & 4.8 & 2 & $0.99^{b}$ & 0.42 & 0.37 & $6,16,4$ \\
\hline US & DRB1 & 5.0 & 3 & 0.69 & 0.45 & 0.32 & ${ }^{\star} 0701,{ }^{*} 0301,{ }^{*} 14,{ }^{*} 12,{ }^{*} 0901, * 1001$ \\
\hline US & DQB1 & 4.1 & 1 & 0.56 & 0.33 & 0.32 & not ${ }^{*} 06$, not *0302 \\
\hline
\end{tabular}

${ }^{a}$ Under the null hypothesis, penetrance for all genotype classes was 0.02, based on the prevalence of psoriasis in the population. Populationbased allele frequencies were used for MBSA calculations and simulations. Only significant results (Monte Carlo rank $1-5$ ) are shown. ${ }^{b}$ Upper bound of penetrance parameter. 'Alleles are listed in the order of the magnitude of their contribution to the lod score, largest contributions first. For consistency, all HLA class I alleles are listed by their serological designation, although HLA-C alleles were determined by DNA typing. When the selected alleles producing the highest lod score yielded the lowest risk, these alleles are designated by the prefix 'not' and the penetrance shown refer to those obtained when the remaining alleles were considered to be the risk group. ${ }^{\text {Under }}$ the null hypothesis, penetrance for all genotype classes was 0.43 , based on the prevalence of psoriasis in our sample. Allele frequencies were used for MBSA calculations and simulations were also based on our sample. Only significant results (Monte Carlo rank $1-5$ ) are shown. 


\section{Population-based}
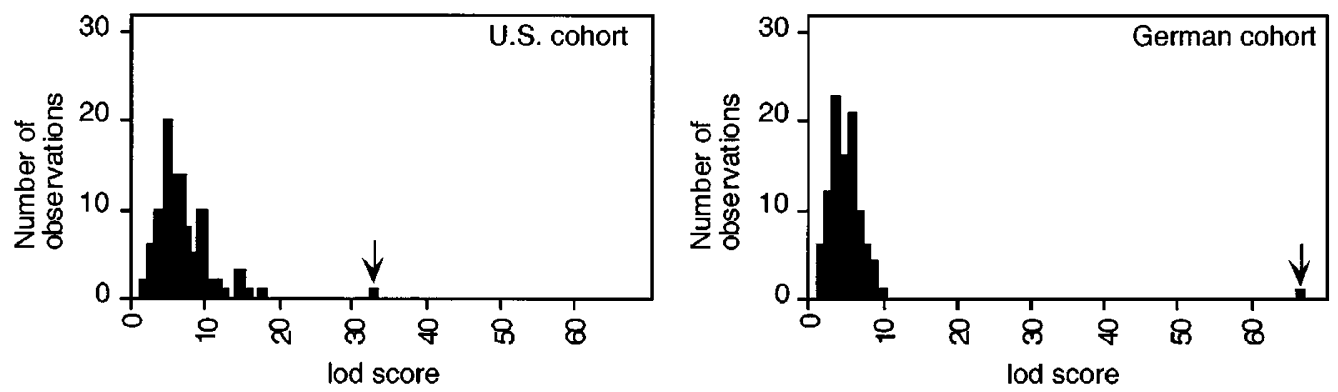

Sample-based
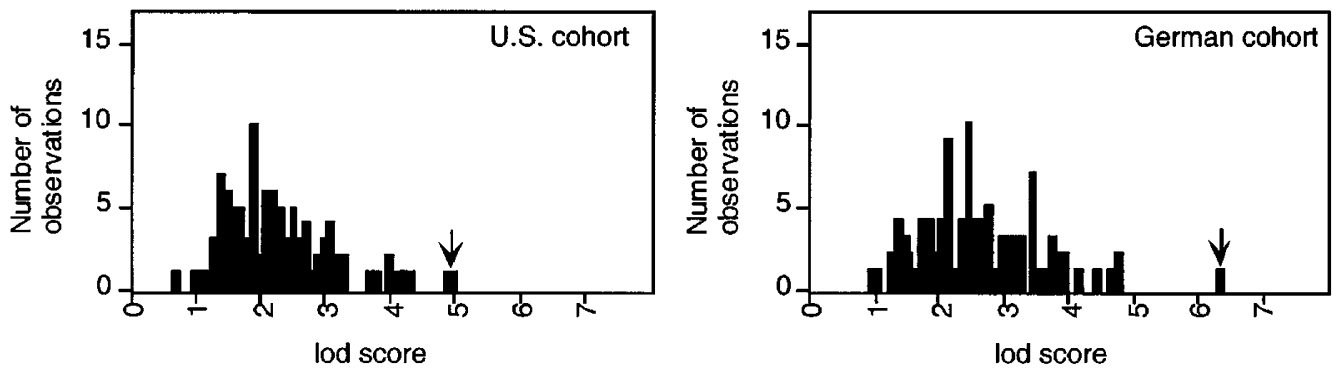

Figure 2 Histogram of MBSA lod score distributions for HLA-C. Results for the population-based and sample-based methods are shown. In each panel, results obtained for 99 genotype simulations and the observed data are shown. Lod scores based on the observed data are indicated by an arrow $(\downarrow)$.

As shown in Table 2, MBSA provides information on genotype-phenotype relationships that is not provided by the TDT. However, segregation analysis is susceptible to ascertainment bias, for which appropriate correction is difficult. $^{35}$ Our sample is inherently biased for multiplex involvement, as all affected individuals in this study had at least one affected relative (Table 1), as opposed to only $10-$ $15 \%$ of all juvenile onset psoriatics in general. ${ }^{39}$ In order to assess the effects of this ascertainment bias, we performed the entire analysis twice. The population-based analysis used prevalence estimates and allele frequencies derived from the general population to define the penetrance parameters and allele frequencies under the null hypothesis of no segregation In the sample-based analysis, these parameters were derived from our own sample. Under either scenario, the MBSA identified HLA alleles belonging to the EH57.1 ancestral haplotype as risk-conferring, and the highest penetrances were associated with HLA-B and -C in both the German and US cohorts (Figure 2 and Table 2). These findings were in excellent agreement with published results. ${ }^{11,12,24,37}$ Indeed, the sample-based analysis demonstrated that the signals of gene transmission contained in the actual pedigree data were sufficiently strong as to identify segregation of a candidate disease allele over the 'noise' introduced by ascertainment bias, which is represented by the use of our own sample to model the null hypothesis.

While both analyses yielded statistically significant results, the penetrance estimates we obtained differed markedly between the two. It is instructive to note that the penetrance of the risk/non-risk class divided by the penetrance of the non-risk/non-risk class is equivalent to the genotypic risk ratio $\gamma$ defined by Risch and Merikangas. ${ }^{3}$ These ratios can be calculated for various loci from the data in Table 2. In a separate set of calculations, we determined that $\gamma=5.1$ in psoriasis (calculations available upon request). These calculations were based on the assumption of Hardy-Weinberg equilibrium, a population frequency of $2 \%$, the control allele frequencies used in this report, and a disease allele frequency predicated on RH1, a PSORS1 risk haplotype that we identified by analysis of recombinant ancestral MHC haplotypes. ${ }^{24}$ Interestingly, this value is very similar to the penetrance ratio of 5.5 calculated for HLA-C in the US population under the population-based model. In contrast, the penetrance ratio was only 1.1 under the sample-based model. In the German population, the penetrance ratio was 16.0 under the population-based model and 1.9 under the sample-based model. The higher values of both penetrance ratios in the German sample may be explained by the higher proportion of extended pedigrees in the German group (Table $1)$, by chance, or by a combination of the two.

We would argue that the population-based MBSA captures the state of nature with greater fidelity than does the samplebased MBSA, because the former makes use of additional, independent information (e.g., population-based allele and disease frequencies). However, the penetrance values obtained under these circumstances can be overestimates, due 
A
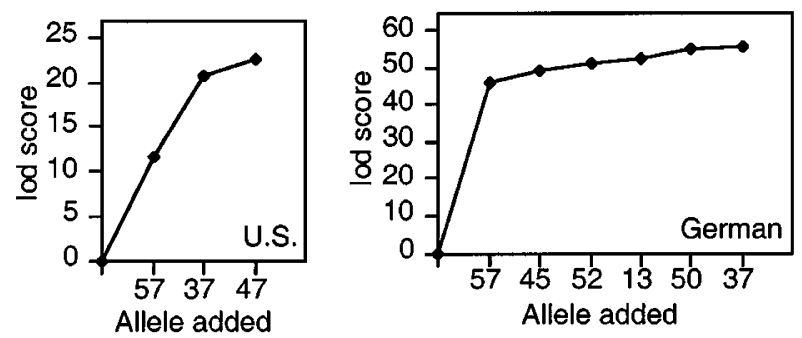

B
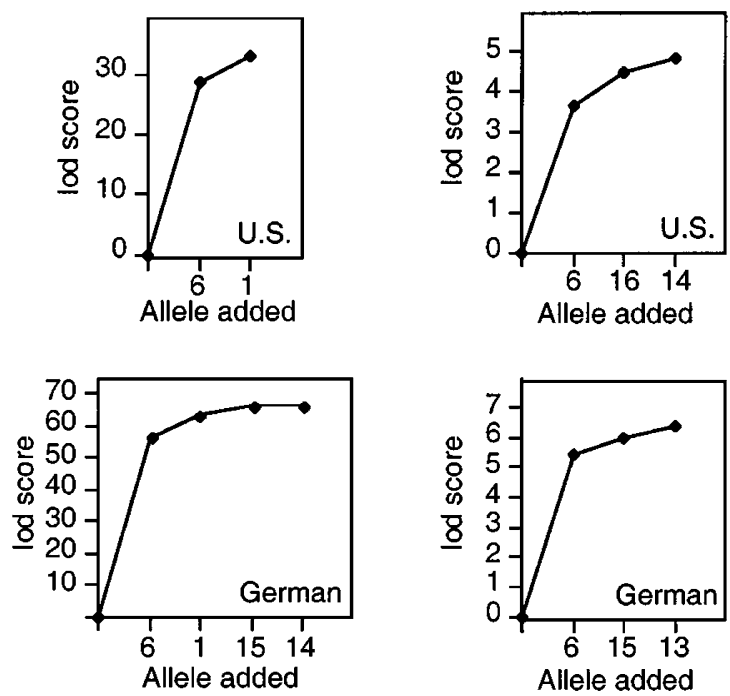

Population-based

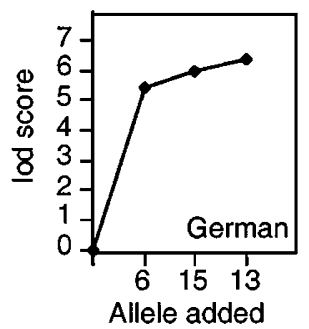

Sample-based

Figure 3 MBSA lod scores obtained as a function of allele added to the risk group. Results for (A) HLA-B and (B) HLA-C are shown. All results shown in (A) derive from the populationbased method, as the sample-based results were not significant.

to deliberate ascertainment of multiplex families. On the other hand, the penetrance values determined by the samplebased method are likely to be underestimates due to inaccurate modeling of the general population (diseased and non-diseased). As in all segregation studies, this dilemma can best be addressed by careful ascertainment of the sample in such a way as to allow conditioning of the data on that scheme. ${ }^{35}$ It would be interesting to compare the performance of MBSA with other methods for analysis of HLA data, such as the haplotype method of Thomson et al. ${ }^{40}$ However, such comparisons are beyond the scope of this manuscript.

Allelic heterogeneity is another aspect of the genotypephenotype relationship made accessible by MBSA. We observed measurable increases in the MBSA lod score as additional risk alleles are added (Figure 3), raising the question of allelic heterogeneity for PSORS1. Our recent studies of PSORS1 indicate that at least $61 \%$ of affected individuals in our sample are identical by descent at this locus. ${ }^{24}$ Therefore, the bulk of risk in our population must be carried by only one allele. However, multiple alleles contribute to the MBSA lod scores, particularly at HLA-A, HLA-B, HLA-DR, and HLADQ (Table 2, Figure 3 ). This finding appears to be explained by the fact that many ancestral recombination events have occurred within HLA since the emergence of modern humans, despite the existence of strong linkage disequilibrium from HLA-A to HLA-DQ. ${ }^{41,42}$ Indeed, each of the HLA-B alleles shown as contributing to the lod score in Figure $3 \mathrm{~A}$ are observed in haplotypic association with HLA-Cw6 in our sample (250/251 occurrences for B57, 56/62 for B37, 15/18 for B45, 6/23 for B52, $9 / 9$ for $\mathrm{B} 50$, and $2 / 3$ for $\mathrm{B} 47$ ). The most parsimonious explanation of our results is that many individuals carry HLA alleles other than those found on EH57.1 as a result of ancestral recombination events, rather than allelic heterogeneity. Indeed, there is a trend toward an increasing number of disease alleles as a function of distance from HLA-C, the locus closest to the probable location of PSORS1 (Table 2). However, MBSA would be well-suited to detect allelic heterogeneity in settings where it truly exists, assuming that the founders are sufficiently distant to assure a sufficient number of observable events.

The MBSA and the TDT share the disadvantage that neither method can be relied upon to identify the true disease allele among a set of positional candidates in strong linkage disequilibrium. As mentioned earlier, we have attempted to overcome this problem through high-density genotyping followed by haplotype construction and cluster analysis of recombinant ancestral haplotypes. ${ }^{24}$ That analysis points to an interval telomeric to HLA-C, and actually appears to exclude HLA-C itself as the PSORS1 gene. The present analysis is consistent with that conclusion, but does not provide additional evidence for it.

We have not attempted to analyse these non-HLA microsatellite markers by MBSA, as they are mainly derived from intergenic regions. However, as the search for causative genes in the 'complex' genetic disorders proceeds into the phase of high-density mapping using single-nucleotide polymorphisms (SNPs), MBSA should be well suited to analysis of SNP haplotypes derived from these genes.

In conclusion, we have shown here that MBSA is capable of providing important information on genotype-phenotype relationships, and on possible allelic heterogeneity. However, this information will need to be carefully interpreted in light of ascertainment bias and possible linkage disequilibrium, and the best results will be obtained when ascertainment is appropriately controlled.

\section{Acknowledgements}

We thank Clauda Gier, Hilke Clasen and Dorit Schuster for assistance with DNA-based HLA typing, Angela Schulz, Corinna Greve, Bettina Marxsen, Marion Schromm and Anke Siemsen for assistance with serological HLA typing, and Margaret Terhune, Chris Bichakjian, and Winfried Lenk for assistance with clinical evaluation. We also thank Wolfgang Müller-Ruchholtz and Martin Krönke for advice and 
support. This research was supported by award DFG-WE 905/1-1 from the German Research Foundation (EW, TH, SJ), by USPHS awards P30 HG00209-03 and R01 AR4274-01 (JTE, SWG, PS, RN) and by R29 GM52205 and R01 GM 56515 (SWG).

\section{References}

1 Altmuller J, Palmer LJ, Fischer G, Scherb H, Wjst M: Genomewide scans of complex human diseases: true linkage is hard to find. Am J Hum Genet 2001; 69: 936-950.

2 Ioannidis JP, Ntzani EE, Trikalinos TA, Contopoulos-Ioannidis DG: Replication validity of genetic association studies. Nat Genet 2001; 29: 306-309.

3 Risch N, Merikangas K: The future of genetic studies of complex human diseases. Science 1996; 273: 1516-1517.

4 Xiong M, Guo SW: The power of linkage detection by the transmission/disequilibrium tests. HumHered 1998; 48:295 - 312.

5 Schaid DJ: Transmission disequilibrium, family controls, and great expectations. Am J Hum Genet 1998; 63: 935 - 941.

6 Spielman RS, Ewens WJ: The TDT and other family-based tests for linkage disequilibrium and association. Am J Hum Genet 1996; 59: 983 - 989 .

7 Ewens WJ, Spielman RS: The transmission/disequilibrium test: history, subdivision, and admixture. Am J Hum Genet 1995; 57 $455-464$.

8 Martin ER, Bass MP, Kaplan NL: Correcting for a potential bias in the pedigree disequilibrium test. Am J Hum Genet 2001; 68 $1065-1067$.

9 Martin ER et al: A test for linkage and association in general pedigrees: the pedigree disequilibrium test. Am J Hum Genet 2000; 67: 146-154.

10 Guo SW, Thompson EA: A Monte Carlo method for combined segregation and linkage analysis. Am J Hum Genet 1992; 51: $1111-1126$.

11 Jenisch $S$ et al: Linkage analysis of HLA markers in familial psoriasis: strong disequilibrium effects provide evidence for a major determinant in the HLA-B/ -C region. Am J Hum Genet 1998; 63: $191-199$.

12 Jenisch S et al: Linkage disequilibrium analysis of familial psoriasis: Identification of multiple disease-associated MHC haplotypes. Tissue Antigens 1999; 53: $135-146$.

13 Vyse TJ, Todd JA: Genetic analysis of autoimmune disease. Cell 1996; 85: $311-318$

14 Elder JT et al: The genetics of psoriasis. Arch Dermatol 1994; 130: 216-224.

15 Tomfohrde $\mathrm{J}$ et al: Gene for familial psoriasis susceptibility mapped to the distal end of human chromosome $17 \mathrm{q}$. Science 1994; 264: 1141 - 1145 .

16 Matthews D et al: Evidence that a locus for familial psoriasis maps to chromosome 4q. Nat Genet 1996; 14: $231-233$.

17 Nair RP et al: Evidence for two psoriasis susceptibility loci (HLA and $17 \mathrm{q})$ and two novel candidate regions (16q and 20p) by genome-wide scan. Hum Mol Genet 1997; 6: 1349-1356.

18 Trembath RC et al: Identification of a major susceptibility locus on chromosome $6 \mathrm{p}$ and evidence for further disease loci revealed by a two stage genome-wide search in psoriasis. Hum Mol Genet 1997; 6: 813-820.

19 Samuelsson L et al: A genome-wide search for genes predisposing to familial psoriasis by using a stratification approach. Hum Genet 1999; 105: 523 -529.

20 Capon $\mathrm{F}$ et al: Searching for psoriasis susceptibility genes in Italy: genome scan and evidence for a new locus on chromosome 1. J Invest Dermatol 1998; 112: 32 - 35

21 Hamosh A, Scott AF, Amberger J, Valle D, McKusick VA: Online Mendelian Inheritance in Man (OMIM). Hum Mutat 2000; 15 $57-61$.

22 Oka A et al: Association analysis using refined microsatellite markers localizes a susceptibility locus for psoriasis vulgaris within a $111 \mathrm{~kb}$ segment telomeric to the HLA-C gene. Hum Mol Genet 1999; 8: $2165-2170$
23 Balendran $\mathrm{N}$ et al: Characterization of the major susceptibility region for psoriasis at chromosome 6p21.3. I Invest Dermatol 1999; 113: 322 - 328 .

24 Nair RP et al: Localization of psoriasis-susceptibility locus PSORS1 to a 60-kb interval telomeric to HLA-C. Am J Hum Genet 2000; 66: $1833-1844$.

25 Elder JT, Voorhees JJ: Psoriasis. in Prinicples of Molecular Medicine, Vol. 1 (ed. Jameson, J.L.) 793-800 (Humana, Totowa, NJ, 1998).

26 Henseler T, Christophers E: Psoriasis of early and late onset: characterization of two types of psoriasis vulgaris. J Am Acad Dermatol 1985; 13: 450-456.

27 Darke C, Dyer P: Clinical HLA typing by cytotoxicity. in Histocompatibility Testing: A Practical Approach, Vol. 1 (eds. Dyer, P. \& Middleton, D.) 51-80 (Oxford Univ. Press, Oxford, 1993).

28 Bunce M. et al: High resolution HLA-C typing by PCR-SSP: identification of allelic frequencies and linkage disequilibria in 604 unrelated random UK Caucasoids and a comparison with serology. Tissue Antigens 1996; 48: 680-691.

29 Kimura A, Sasazuki T: Eleventh International Histocompatibility Workshop reference protocol for the HLA DNA-typing technique; in HLA 1991; Tsuji K, Aizawa M, Sasazuki T (eds). Proceedings of the Eleventh International Histocompatibility Workshop and Conference, Vol. 1 (Oxford, New York, Tokyo: Oxford University Press, 1993). pp. $397-419$.

30 Tsuji K, Aizawa M, Sasazuki T. (eds). HLA 1991: Proceedings of the Eleventh International Histocompatibility Workshop and Conference, 1220 Oxford: Oxford University Press, 1992.

31 Elston RC, Stewart J: A general model for the genetic analysis of pedigree data. Hum Hered 1971; 21: 523-542.

32 Lange K, Weeks D, Boehnke M: Programs for Pedigree Analysis: MENDEL, FISHER, and dGENE [letter]. Genet Epidemiol 1988; 5: $471-472$.

33 Boehnke M: Estimating the power of a proposed linkage study: a practical computer simulation approach. Am J Hum Genet 1986; 39: $513-527$

34 Christophers E, Sterry, W: Psoriasis; in Dermatology in General Medicine; (Fitzpatrick TB, Eisen AZ, Wolff K, Freedberg IM, Austen KF (eds)) New York: McGraw-Hill, 1993, pp. 489-514.

35 Elston RC: 'Twixt cup and lip: how intractable is the ascertainment problem? Am J Hum Genet 1995; 56: 15 - 17 .

36 Degli Esposti MA, Leelayuwat C, Dawkins RL: Ancestral haplotypes carry haplotypic and haplospecific polymorphisms of BAT1: possible relevance to autoimmune disease. Eur J Immunogenet 1992; 19: $121-127$

37 Schmitt-Egenolf M, Eiermann TH, Boehncke WH, Ständer M, Sterry W: Familial juvenile onset psoriasis is associated with the human leukocyte antigen (HLA) class I side of the extended haplotype Cw6- B57-DRB1*0701-DQA1*0201-DQB1*0303: a population- and family-based study. I Invest Dermatol 1996; 106: $711-714$.

38 George VT, Elston RC: Testing the association between polymorphic markers and quantitative traits in pedigrees. Genet Epidemiol 1987; 4: $193-201$.

39 Christophers E, Henseler T: Psoriasis type I and type II as subtypes of nonpustular psoriasis. in Psoriasis Roenigk $\mathrm{H}$, Maibach, H, (eds) 15-21 New York: Marcel Dekker 1990.

40 Thomson G et al: Genetic heterogeneity, modes of inheritance, and risk estimates for a joint study of Caucasians with insulindependent diabetes mellitus. Am J Hum Genet 1988; 43: 799_ 816.

41 Martin M, Mann D, Carrington M: Recombination rates across the HLA complex: use of microsatellites as a rapid screen for recombinant chromosomes. Hum Mol Genet 1995; 4: 423-428.

42 van Endert PM, Lopez MT, Patel SD, Monaco JJ, McDevitt HO: Genomic polymorphism, recombination, and linkage disequilibrium in human major histocompatibility complex-encoded antigen-processing genes. Proc Natl Acad Sci USA 1992; 89: $11594-11597$. 